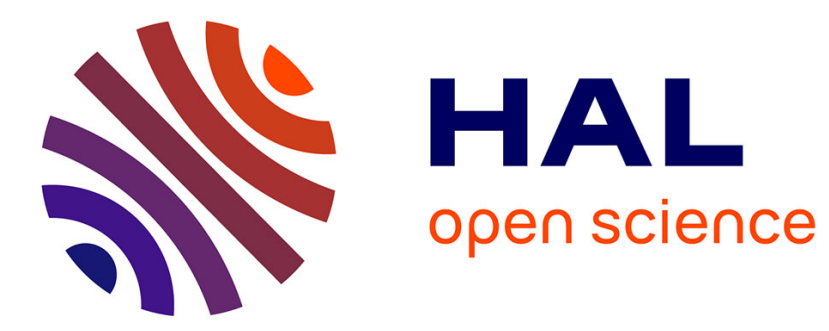

\title{
Un patron peut en cacher un autre
}

Nicolas Jounin, Louise Paternoster

\section{To cite this version:}

Nicolas Jounin, Louise Paternoster. Un patron peut en cacher un autre. Savoir / Agir , 2009, 10 (4), pp.13-22. 10.3917/sava.010.0013 . hal-01822194

\section{HAL Id: hal-01822194 https://hal.science/hal-01822194}

Submitted on 16 Aug 2018

HAL is a multi-disciplinary open access archive for the deposit and dissemination of scientific research documents, whether they are published or not. The documents may come from teaching and research institutions in France or abroad, or from public or private research centers.
L'archive ouverte pluridisciplinaire HAL, est destinée au dépôt et à la diffusion de documents scientifiques de niveau recherche, publiés ou non, émanant des établissements d'enseignement et de recherche français ou étrangers, des laboratoires publics ou privés. 


\section{UN PATRON PEUT EN CACHER UN AUTRE}

Sous-traitance et intérim dans les secteurs du bâtiment et du nettoyage Nicolas Jounin, Louise Paternoster

\section{Editions du Croquant | « Savoir/Agir »}

2009/4 $\mathrm{n}^{\circ} 10 \mid$ pages 13 à 22

ISSN 1958-7856

ISBN 9782914968676

Article disponible en ligne à l'adresse:

https://www.cairn.info/revue-savoir-agir-2009-4-page-13.htm

\section{Pour citer cet article :}

Nicolas Jounin, Louise Paternoster « Un patron peut en cacher un autre. Soustraitance et intérim dans les secteurs du bâtiment et du nettoyage », Savoir/Agir 2009/4 (n 10), p. 13-22.

DOI 10.3917/sava.010.0013

Distribution électronique Cairn.info pour Editions du Croquant.

(C) Editions du Croquant. Tous droits réservés pour tous pays.

La reproduction ou représentation de cet article, notamment par photocopie, n'est autorisée que dans les limites des conditions générales d'utilisation du site ou, le cas échéant, des conditions générales de la licence souscrite par votre établissement. Toute autre reproduction ou représentation, en tout ou partie, sous quelque forme et de quelque manière que ce soit, est interdite sauf accord préalable et écrit de l'éditeur, en dehors des cas prévus par la législation en vigueur en France. Il est précisé que son stockage dans une base de données est également interdit. 


\section{Un patron peut en cacher un autre Sous-traitance et intérim dans les secteurs du bâtiment et du nettoyage}

NiCOLAS JOUNIN, sociologue, université Paris 8.

ET LOUISE PATERNOSTER, sociologue.

1. Alain Supiot, Critique $d u$ droit $d u$ travail, Paris, PUF, 2002, première éd. 1994.
On n'est pas des patrons » : c'est ainsi que le PDG $\checkmark$ d'une entreprise sous-traitante de ferraillage, dans le secteur du bâtiment, parle de lui-même et de ses homologues. "On n'est pas des patrons », argumente-t-il, parce que ces anciens cadres devenus patrons à la faveur de l'externalisation de leur métier manquent de compétences ; et parce qu'ils disposent de bien peu de marge de manœuvre, face aux exigences de leurs commanditaires, dans la gestion de leur entreprise et de leur personnel. Ils sont pourtant, du point de vue du droit, les incontestables patrons des ferrailleurs des chantiers, tandis que les clients ne sont «que » les bénéficiaires de la prestation - des extorqueurs lointains de la plus-value réalisée sur le travail des ouvriers.

La loi de 1898 sur les accidents du travail est considérée comme fondatrice du droit du travail en ce qu'elle distingue une nouvelle forme de contrat, où l'un des deux contractants met à disposition sa force de travail, c'est-àdire sa liberté et sa volonté, pendant un temps déterminé. La subordination est consacrée et dans le même temps limitée par le droit, qui précisera les limites du pouvoir de l'employeur'. Cette idée d'un commandement direct répond au développement de la grande firme taylorienne, qui devient au $\mathrm{xx}^{\mathrm{e}}$ siècle la représentation sinon la réalité dominante du monde du travail. L'interdiction du « marchandage » (ici la fourniture de main-d'œuvre à but lucratif), formulée de diverses manières entre 1848 et 1973, en est le complément, puisqu'elle restreint théoriquement le recours à des entrepreneurs intermédiaires.

Les choses changent cependant à partir des années 1970. En 1973, le code des marchés publics cesse d'exiger une 
autorisation préalable pour sous-traiter : s'enclenche une dynamique de privatisation "par en bas ", sans passer par des changements de statut des institutions concernées. En 1975, la loi sur la sous-traitance, tout en cherchant à sécuriser l'opération, ne lui pose que très peu de limites. C'est une forme juridique hybride qui se dessine, puisque le contrat de sous-traitance se situe entre le contrat de vente et le contrat de travail. La doctrine, puis la jurisprudence conviennent que le sous-traitant se distingue du pur fournisseur en ce qu'il obéit à des directives, des schémas conçus par son client, tout en gardant une certaine autonomie, faute de quoi on bascule dans le droit du travail ${ }^{2}$. Par la loi du 3 janvier 1972, un an avant celle réinterdisant le marchandage, les agences d'intérim, jusque-là illégales, s'en voient confier le monopole 3 .

Certes, autour de ces dispositifs se commettent nombre d'illégalités. Mais il serait vain de réduire les stratégies patronales à ces dernières, ou d'identifier le droit à des règles incantatoires et des garde-fous inutiles. Les stratégies patronales prennent appui sur le droit pour se déployer et s'adaptent à ses transformations, éventuellement après les avoir influencées. Les stratégies d'externalisation s'interprètent notamment comme un moyen de contourner des règles (le droit du travail) grâce à d'autres (le droit commercial) : les entreprises commanditaires recourent au contrat d'entreprise pour se débarrasser du contrat de travail, dont la rupture est plus difficile. Cette conversion juridique a son pendant dans l'organisation des entreprises : ce ne sont plus les directions des ressources humaines qui sont concernées par le personnel externalisé, mais les directions des achats qui, entre deux commandes de produits, négocient les contrats de soustraitance et d'intérim.

Néanmoins, cela ne signifie pas l'élimination pure et simple des contrats de travail, comme dans le cas des "faux indépendants », mais un report de leur gestion sur des employeurs intermédiaires. Ces derniers mettent en place une gestion plus flexible, plus brutale, en partie parce qu'ils bénéficient de législations spécifiques qui leur confèrent moins d'obligations en matière de relations de travail, mais surtout parce qu'ils commettent des illégali-
2. J.-P. Babando, $L a$ sous-traitance dans la construction, Paris, LexisNexis, Paris, 2005.

3. A. Morice, "Quand la lutte contre l'emploi illégal cache les progrès de la précarité légale », in D. Fassin, A. Morice, C. Quiminal (dir.), Les politiques de l'immigration à l'épreuve des sans-papiers, Paris, La Découverte, 1997, p. 177-196. 
tés que leurs commanditaires ne voudraient ou ne pourraient assumer.

Dans cet article, nous revenons donc sur ces situations où l'employeur officiel (sous-traitant ou agence d'intérim), auquel le salarié est subordonné, est lui-même dépendant d'un donneur d'ordres ; situations hybrides, où l'identité du patron est incertaine. Se demander alors qui est le patron, n'est pas un simple jeu de l'esprit, mais une préoccupation concrète pour les travailleurs concernés, dont les solutions dépassent le cadre d'analyse traditionnel de la « relation triangulaire».

Nous aborderons ensemble sous-traitance et intérim, les considérant comme deux variantes de la figure de l'employeur intermédiaire. Certes, les deux sont théoriquement et juridiquement distincts, la sous-traitance portant théoriquement sur un métier, un produit ou un savoir-faire particuliers, tandis que l'intérim se consacrerait à une pure fourniture de main-d'œuvre. Mais que penser quand le DRH d'une grande entreprise soustraitante déclare que "notre savoir-faire, c'est justement le management de personnels faiblement qualifiés », ou quand, à l'inverse, des agences d'intérim se spécialisent sur certains métiers? Nous évoquerons deux secteurs : le bâtiment (plus précisément le gros œuvre et le nettoyage ${ }^{4}$, tous deux en région parisienne). Nous reviendrons sur les formes et les effets du dédoublement du patron dans le temps "normal », quotidien, du travail, puis dans le temps suspendu, extraordinaire, celui du conflit : la grève.

\section{À froid}

Un jour d'hiver, sur un bâtiment en construction, il pleut. Faut-il déclarer le chantier en intempéries ? La direction du chantier veut que l'on travaille. Les ouvriers

4. Nicolas Jounin, Chantier interdit an public, Paris, La Découverte, 2008 ; Louise Paternoster, Pratiques patronales et résistances ouvrières dans la production de propreté, mémoire de M2, ENSEHESS, 2009. refusent, ou plutôt une partie d'entre eux : les salariés embauchés de l'entreprise générale, ceux qui appartiennent à la même entreprise que la direction. Derrière, dans une réserve prudente, les personnels sous-traitants et intérimaires attendent l'issue du combat. Plutôt favorables à la revendication des autres ouvriers, mais attentistes. L'entreprise sous-traitante en ferraillage, pressée par la 
direction du chantier, exhorte ses ouvriers à se mettre au travail. Ces derniers, tiraillés entre la solidarité avec les embauchés de l'entreprise générale et la peur des représailles - ils sont non seulement sous-traitants mais aussi intérimaires -, finissent par obtempérer.

L'épisode n’a rien d'étonnant. Et pourtant, rapporté à la configuration juridique du chantier, il devrait nous étonner : ce sont précisément les travailleurs qui ne sont pas liés par un contrat de travail avec l'entreprise générale, ceux qui ne lui sont pas formellement subordonnés, qui lui sont le plus soumis. Entre les deux pôles s'intercale l'entreprise sous-traitante et, entre elle et sa commanditaire, un contrat commercial. Un tel contrat est censé être plus égalitaire que le contrat de travail, tandis que les obligations du sous-traitant, limitées à l'accomplissement de certaines tâches prévues par avance dans le cahier des charges, sont théoriquement plus restreintes que celles d'un salarié, supposé obéir sur le moment. Dans la pratique du chantier, la situation prend une autre tournure : la soumission des ouvriers sous-traités est renforcée. Ces derniers endossent, en plus de leur propre subordination à leur employeur, la dépendance commerciale de ce dernier. Si le client juge la prestation décevante, son mécontentement risque de se reporter rapidement sur les salariés du fournisseur, par le biais d'avertissements voire de licenciements.

Cependant, l'employeur intermédiaire ne représente pas uniquement, pour le commanditaire, une garantie que ses consignes seront plus efficacement suivies qu'avec ses propres salariés. Il n'est pas seulement son allié mais aussi, malgré l'asymétrie, son concurrent dans la captation de la plus-value réalisée sur le travail des salariés. Cette tension se manifeste initialement dans la négociation du contrat ; elle parcourt ensuite le quotidien de son exécution. Le cahier des charges peut être interprété, contourné, voire transgressé : l'intérêt du sous-traitant est d'en faire moins que ce sur quoi il s'est engagé, celui du commanditaire d'en avoir le plus possible pour son argent. À partir de ce canevas se tisse un jeu de faux dupes, dans la mesure où chacun sait que le tarif négocié interdit généralement un respect parfait du cahier des charges. Le dédoublement de l'employeur suscite entre le donneur d'ordres et son 
sous-traitant des liens de solidarité mais aussi des antagonismes.

Une telle situation multiplie les injonctions contradictoires pesant sur les salariés : ils peuvent être accusés aussi bien de ne pas faire le travail, que de ne pas avoir masqué le travail pas ou mal fait ou encore d'en faire trop pour le client. La commercialisation d'activités, à travers la sous-traitance, permet en effet de rééditer plus souvent la course au moins disant: dans le nettoyage, tous les trois ou cinq ans, il se trouve toujours un nouveau prestataire pour rafler le marché à un prix rabaissé. Le faible développement du machinisme, commun aux deux secteurs, entraîne le report de ces baisses sur le coût du travail. Cela passe par une "rationalisation » du travail, une intensification de la discipline, des licenciements, des reclassements contraints... Pratiquement, la rationalisation prend la forme d'économies de bouts de chandelles : on économise sur les produits, les protections, etc. Dans un contexte de pénurie de moyens humains et matériels programmée, la «triche » obligatoire transforme les soustraitants en éternels fautifs ; elle renforce et justifie la méfiance et le contrôle du donneur d'ordres à leur égard.

L'employeur intermédiaire est ainsi à l'avant-poste des contradictions de l'externalisation, poursuivant ses propres intérêts tout en déplaisant le moins possible à son commanditaire. Toutefois, quoiqu'il apparaisse juridiquement comme le nœud de la relation dite «triangulaire ", son rôle dans la pratique est parfois plus secondaire. Lorsqu'il se contente de jouer le rôle d'interface, les contradictions sont aplanies. Dans le bâtiment, on trouve ainsi de nombreux intérimaires qui dépendent, pratiquement et effectivement, de chefs de chantier ou d'entreprises utilisatrices, tandis que les agences d'intérim ne sont que les dispositifs par lesquels cette dépendance transite et prend forme. Aussi les agences ne fournissent-elles pas toujours de la main-d'œuvre, contrairement à leur fonction officielle, se contentant d'octroyer un statut précaire à une maind'œuvre déjà sélectionnée par les utilisateurs.

Dans le nettoyage, c'est une spécificité conventionnelle qui fait du sous-traitant le maillon faible de la relation : l'annexe 7 de la convention collective spécifie que 
l'entreprise obtenant un marché de nettoyage doit également reprendre le personnel correspondant. Soulignons que cette disposition, pour protectrice des salariés qu'elle puisse paraître, a été obtenue par le patronat du secteur qui refusait de s'embarrasser de salariés devenus surnuméraires suite à la perte d'un contrat, autrement dit d'assumer l'ensemble des prérogatives et des obligations classiques de l'employeur. Cette disposition favorise l'ancienneté des salariés sur le site de travail. Ils sont installés dans un faceà-face durable avec le commanditaire tandis que le soustraitant, qui fait théoriquement le lien entre les deux, est le moins stable des protagonistes, se réduisant à une interface. Ce faisant, les salariés adhèrent parfois davantage aux objectifs du donneur d'ordres qu'à ceux du sous-traitant, comme dans cet hôpital où une ouvrière du nettoyage signale à la cadre de santé les insuffisances de ce dernier et se voit réprimandée par son employeur. De telles situations permettent de comprendre pourquoi des sociétés soustraitantes interdisent tout contact entre leurs salariés et des représentants du donneur d'ordres. Elles tentent ainsi de réinstaurer un certain contrôle sur le travail (et limiter les risques de discrédit). Néanmoins ce n'est pas toujours possible, car le travail peut appeler une coopération et donc une communication quotidiennes, qui passe par des consignes, des ordres, voire des insultes ou du harcèlement de la part de la hiérarchie de l'entreprise utilisatrice. Dès lors, en maintes occasions, le recours à des employeurs intermédiaires permet au donneur d'ordres de concentrer les prérogatives du patron sans s'embarrasser des contreparties. Dans l'intérim du bâtiment, il suffit de signifier le jour même à l'agence qu'on ne veut plus de tel salarié pour que le contrat de ce dernier prenne illégalement fin. Même dans le cadre de la sous-traitance, il est courant que le commanditaire demande le transfert ou le licenciement d'un ouvrier sous-traitant.

En sens inverse, les salariés externalisés peuvent faire pression sur le donneur d'ordres pour la sélection de l'employeur intermédiaire. Il arrive ainsi que les intérimaires du bâtiment refusent un changement d'agence d'intérim imposé par l'entreprise utilisatrice, parce que l'agence est trop loin, ou a la réputation de commettre des vols sur les fiches de 
paie. Dans le nettoyage, lors du renouvellement du marché, le sous-traitant peut ne plus être seulement l'intermédiaire, mais devenir l'objet d'une négociation directe entre les salariés et le commanditaire. La responsable des achats d'un hôpital explique ainsi : "C'est toujours la grande angoisse pour eux. Ils sont sans cesse derrière moi à me faire une pression pas possible. "Faites attention", "prenez pas toujours le moins cher", enfin vous voyez ils me donnent des conseils, je n'en tiens pas compte, bien sûr, mais ils sont inquiets. » Bien évidemment, ces demandes sont bien moins suivies que lorsqu'un donneur d'ordres réclame le licenciement d'un salarié sous-traitant ou intérimaire. Néanmoins, dans le secteur du nettoyage, en raison de la configuration syndicale, elles ne sont pas complètement inefficaces : même si la pratique syndicale est marquée par des formes de collusions, elle implique en retour la possibilité de faire pression au moment de l'appel d'offres.

\section{À chaud}

Une journée de printemps 2006, les rues et les amphithéâtres se font l'écho de la révolte des étudiants et travailleurs. Dans le chantier de nettoyage de la gare RER près d'une des universités mobilisées, l'appel d'offres vient de passer. À l'occasion, trois salariés sont licenciés, la prime de travail nocturne retirée des fiches de paie. Les salariés réagissent immédiatement : ils décident la «levée du chantier ", se mettent en grève et occupent le local de nettoyage. Pour accroître la gêne, ils éventrent les poubelles, éparpillent les papiers. Les cadres de la SNCF et du sous-traitant en nettoyage se succèdent pour tenter, au fur et à mesure, d'essuyer les traces de la lutte. Les deux entreprises se renvoient la responsabilité : la société intermédiaire se présente comme "victime » du budget « intenable » de la SNCF, la direction de celle-ci dit ne pas être concernée par les agissements de ses sous-traitants.

En divisant les collectifs de salariés, en démultipliant les employeurs sur un même site, la sous-traitance et l'intérim réduisent la possibilité d'une contestation organisée. Quand malgré tout des conflits se déclarent, le dédoublement du patron constitue un défi pour les stratégies 
syndicales. Vers qui doit se tourner l'action ? Qui détient le pouvoir (celui consacré par la loi, celui qui pourrait l'avoir, et le pouvoir effectif) ?

Première étape, trouver le patron : il arrive que l'employeur intermédiaire soit introuvable, matérialisé par une simple boîte aux lettres, si bien qu'il faut s'attaquer à son commanditaire. Mais ce dernier est alors susceptible de se défausser, arguant qu'il n'a pas de rapport salarial avec les travailleurs puisqu'il était simplement lié par un contrat commercial avec l'intermédiaire fantôme. Si cette configuration est typique de la sous-traitance en cascade, elle se retrouve aussi sous une forme particulière avec l'intérim. La grève de travailleurs sans papiers s'y est brutalement confrontée, puisque la régularisation qui en était la revendication principale devait obligatoirement être parrainée par un patron, ce qui obligeait à localiser ce dernier. La stratégie des grévistes et de leurs soutiens a oscillé entre la mise en cause des agences d'intérim et celle des entreprises utilisatrices. Les premières étaient ciblées en tant qu'employeur direct, mais prétendaient que sans les commandes de donneurs d'ordres elles ne pourraient jamais fournir les promesses d'embauches nécessaires à la régularisation. Les secondes répliquaient qu'elles n'embauchaient pas de sans-papiers et n'étaient pas responsables des errements de leurs fournisseurs ; elles se heurtaient toutefois au constat que nombre d'intérimaires leur étaient subordonnés de longue date, ce qui les plaçait sous la menace d'être reconnues comme le véritable employeur.

Sans prétendre nécessairement qu'il est le « vrai » patron, les salariés du sous-traitant peuvent prendre à partie le donneur d'ordres, en tant que client susceptible de revoir ses exigences à l'égard de son fournisseur. Dans le nettoyage, les salariés peuvent même inciter le client à rompre le contrat avec leur employeur, puisque la convention collective procure à la fois une garantie relative d'être maintenu sur le site et une ancienneté des relations avec le client. Mais les rôles dévolus à chacun des acteurs patronaux restent obscurs. Dans cette zone d'incertitude, le client et le sous-traitant tendent alors à rejeter l'un sur l'autre les responsabilités d'employeur. Cela n'exclut pas derrière ces apparences une solidarité entre les deux 
« patrons », qui passe notamment par une indifférence complice du donneur d'ordres à l'égard des plaintes des personnels externalisés.

Le donneur d'ordres lui-même alterne, suivant les moments, les postures de client victime et de patron mécontent. C'est dans un premier temps en se prévalant de son statut de client qu'il peut chercher en toute légalité à briser la grève. En effet, pendant que le sous-traitant met en œuvre à l'égard de ses salariés une stratégie de répression classique, bridée par l'interdiction légale de remplacer des grévistes, le donneur d'ordres peut se contenter de faire valoir son droit (commercial) à remplacer un fournisseur défaillant par un autre. Cette éventualité constitue pour l'employeur intermédiaire une réelle menace et, en même temps, un prétexte vis-à-vis de ses salariés pour contenir leurs revendications. Quoiqu'elles se situent sur des registres différents et en partie antagonistes, les réactions du donneur d'ordres et de son sous-traitant convergent donc vers la répression de la grève. Le dédoublement du patron ne nuit pas à la solidité de la riposte patronale, bien au contraire.

Face à cette duplicité, la stratégie syndicale peut alors « relocaliser » et réunifier l'autorité patronale, en visant le donneur d'ordres. Au maximum, il s'agit de faire reconnaître par le droit l'existence d'un contrat de travail direct entre le donneur d'ordres et le travailleur sous-traité et intérimaire, et par conséquent réduire la relation triangulaire à une classique relation entre patron et salarié. Au minimum, il s'agit de contraindre le donneur d'ordres à s'asseoir à la table des négociations, par exemple par l'occupation du chantier voire du siège social. En occupant, on désigne un interlocuteur et on cherche à faire reconnaître la validité de cette désignation. Dans les cas connus dans le nettoyage et dans le bâtiment, et alors que le cadre juridique demeure lâche, les occupations de chantiers par les salariés de sous-traitants se sont soldées par des résultats divers, depuis des actions couvertes par la justice jusqu'à des évacuations musclées. Entre ce minimum et ce maximum, on trouve une palette de situations et de stratégies. Ainsi, sur le chantier de l'université Paris 8 - Saint-Denis, une récente grève des ouvriers du nettoyage avait pour 
objet de transférer certaines prérogatives patronales, en particulier la capacité à déterminer le lieu de travail, de l'employeur sous-traitant vers l'université.

Le donneur d'ordres ne se fait pas patron seulement, comme dans le cas cité, lorsque les salariés le lui demandent. Ainsi, dans le temps de l'après-grève, il est susceptible de réclamer au sous-traitant la «tête » de grévistes ou de représentants syndicaux. Alors que pendant le temps de la grève il se retranchait derrière son statut de client, il accapare alors des leviers de pouvoir typiquement patronaux.

\section{Conclusion}

"Ce n'est pas parce que les fonctions patronales sont séparées entre plusieurs individus et entreprises qu'employeurs et entreprises n'existent plus. De même qu'on parle de travailleur collectif on pourrait peut-être aussi parler d'employeur collectif ", écrivait déjà Sami Dassa il y a plus de vingt ans ${ }^{5}$. Cette réalité socioéconomique de mieux en mieux connue peine à trouver sa traduction juridique. En effet, la reconnaissance de la diffusion formelle du pouvoir de commandement - qui n'est pas contradictoire avec l'accroissement de la puissance des donneurs d'ordres, conçue comme capacité à faire valoir leurs intérêts -, se heurte aux formes d'«imputation de responsabilité » établies par le droit du travail : en dehors de pistes récentes ouvertes par certains arrêts, la justice continue de chercher le patron individuel qui devra assumer les obligations de l'employeur'. Face à ces politiques, les ressources et registres d'action des ouvriers varient en fonction de l'organisation du travail. Dans le nettoyage, les travailleurs, relativement stables, nouent entre eux des liens durables qui permettent un ancrage du collectif en dépit des changements d'employeur. Dans le BTP, les effectifs et les collectifs sont plus mouvants, ce qui peut expliquer une moindre conflictualité. Ainsi, paradoxalement, c'est là où l'employeur intermédiaire s'assume le moins comme employeur que la résistance des salariés est la plus forte.
5. Sami Dassa, « L’emploi, enjeux économiques et sociaux. Colloque de Dourdan II ", in Le travail et sa sociologie, Paris, L'Harmattan, 1985, p. 180-187.

6. Elsa Peskine, Réseaux d'entreprise et droit $d u$ travail, Paris, LGDJ, 2008. 